\title{
Optically Controlled Reconfigurable Antenna Array Based on E-Shaped Elements
}

\author{
Arismar Cerqueira Sodré Junior, ${ }^{1}$ Igor Feliciano da Costa, ${ }^{1,2}$ \\ Leandro Tiago Manera, ${ }^{3}$ and José Alexandre Diniz ${ }^{3}$ \\ ${ }^{1}$ Laboratory WOCA (Wireless and Optical Convergent Access), National Institute of Telecommunications (INATEL), \\ João de Camargo Avenue 510, P.O. Box 05-37540-000, Santa Rita do Sapucaí, MG, Brazil \\ ${ }^{2}$ Federal University of Itajubá (UNIFEI), Benedito Pereira dos Santos Avenue 1303, P.O. Box 50-37500-903, Itajubá, MG, Brazil \\ ${ }^{3}$ State University of Campinas (Unicamp), 13083-870 Campinas, SP, Brazil
}

Correspondence should be addressed to Igor Feliciano da Costa; igorfelicianocosta@gmail.com

Received 14 December 2013; Revised 12 March 2014; Accepted 27 March 2014; Published 27 April 2014

Academic Editor: Mohammed Al-Husseini

Copyright (C) 2014 Arismar Cerqueira Sodré Junior et al. This is an open access article distributed under the Creative Commons Attribution License, which permits unrestricted use, distribution, and reproduction in any medium, provided the original work is properly cited.

This work presents the development of optically controlled reconfigurable antenna arrays. They are based on two patch elements with E-shaped slots, a printed probe, and a photoconductive switch made from an intrinsic silicon die. Numerical simulations and experiments have been shown to be in agreement, and both demonstrate that the frequency response of the antenna arrays can be efficiently reconfigured over two different frequency ISM bands, namely, 2.4 and $5 \mathrm{GHz}$. A measured gain of $12.5 \mathrm{dBi}$ has been obtained through the use of two radiating elements printed in a low-cost substrate and a dihedral corner reflector.

\section{Introduction}

Current telecommunications networks require an efficient use of the available spectrum to meet the increasing capacity demand in the optical and wireless sectors as well as the need for energy consumption reduction. Cognitive radio (CR), radio over fiber (RoF), and reconfigurable antenna technologies have independently been utilized to fulfill the changing requirements of modern telecommunications networks. CR is able to automatically detect available frequency channels in a wireless spectrum and adapt transmission parameters, enabling different systems to operate concurrently [1]. CR uses a number of technologies, including adaptive radio, in which the communication system monitors and modifies its own performance, and software defined radio (SDR), in which traditional hardware components, including mixers, modulators, and amplifiers, are configured using advanced pieces of software [2].

RoF technology plays an important role in the integration of wireless and fiber-optic networks [3, 4]. Taking advantage of the benefits of optical fibers in conjunction with the mobility and ubiquity of wireless networks, RoF systems connect a central office (CO) to a base station (BS) by using optical links to transmit one or multiple analogous RF signals without using optical-electronic $(\mathrm{O} / \mathrm{E})$ and electronic-optical (E/O) converters. On the BS side, the signals are recovered by photodetectors and then routed to the remote antenna unit (RAU), which is simple and cost effective because most of the processing components are centralized in the CO. RoF is an essential technology for the provision of untethered access to broadband wireless communications in a range of applications including last mile solutions, extension of existing radio coverage and capacity, backhaul, in-building coverage, outdoor cellular systems, and fixed or mobile broadband wireless access.

Reconfigurable antennas have exhibited great promise for the next generation of wireless systems [5]. This new class of antennas enables reconfigurability for not only the bandwidth but also the radiation pattern and polarization; this reconfiguration is accomplished by using frequencyagile, software-defined, and cognitive radios to cope with extendable and reconfigurable multiservice, multistandard, 


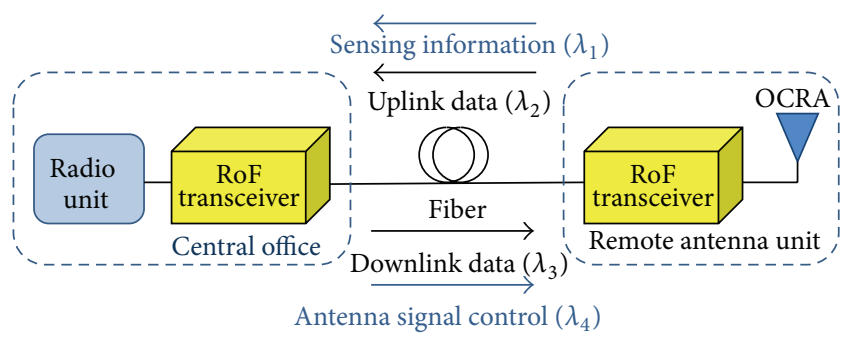

FIgURE 1: Schematic of OCRA implementation.

and multiband operation, and with efficient spectrum and power utilization. These new functionalities can efficiently reduce the number of components, hardware complexity, and cost compared with current radio technology, which relies on incompatible communications systems with inflexible hardware. There are many methods and mechanisms for reconfiguring the antenna structure and/or function [5]. The methods can be based on the integration of radiofrequency microelectromechanical systems (RF-MEMS), PIN diodes, varactors, and photoconductive elements, the physical alteration of the antenna radiating structure, or the use of smart materials such as ferrites and liquid crystal. Particularly, optically controlled reconfigurable antennas (OCRAs) have been proposed and exploited in recent years due to their unique advantages, such as [6-8] easy integration into optical systems; absence of bias lines; elimination of unwanted interference; losses and radiation pattern distortion; reliable reconfiguration mechanisms, especially compared with RFMEMS; linear behavior and activation without producing harmonics; and intermodulation.

This work presents the development of OCRA arrays based on E-shaped elements and an example application that utilizes them, as described in Figure 1. Different wavelengths $(\lambda)$ are used for simultaneously enabling data transmission, monitoring frequency spectrum, and antenna control. Initially, broadband and/or multiband antennas perform spectrum sensing (SS) to discover possible spectrum opportunities. This information is transmitted using RoF links and processed in the $\mathrm{CO}$, which remotely decides how to proceed and optically controls the antenna characteristics. This technique aims to jointly apply CR, RoF, and reconfigurable antenna technologies to harmonically integrate high capacity optical backhaul with energy- and spectrum-efficient wireless access networks by exploiting new spectral resources.

In this way, the proposed OCRA array can be easily integrated into optical networks by using RoF technology to provide multiband and/or multitechnology broadband operation for last mile applications. We have very recently reported a successful implementation of an innovative and cognitive optical wireless network using an OCRA [9]. The proposed architecture allowed performing spectrum sensing and dynamic resource allocation to dynamically adapt the frequency band and/or antenna radiation pattern as a function of the wireless environment. Experimental results have demonstrated the use of the OCRA, and implementing
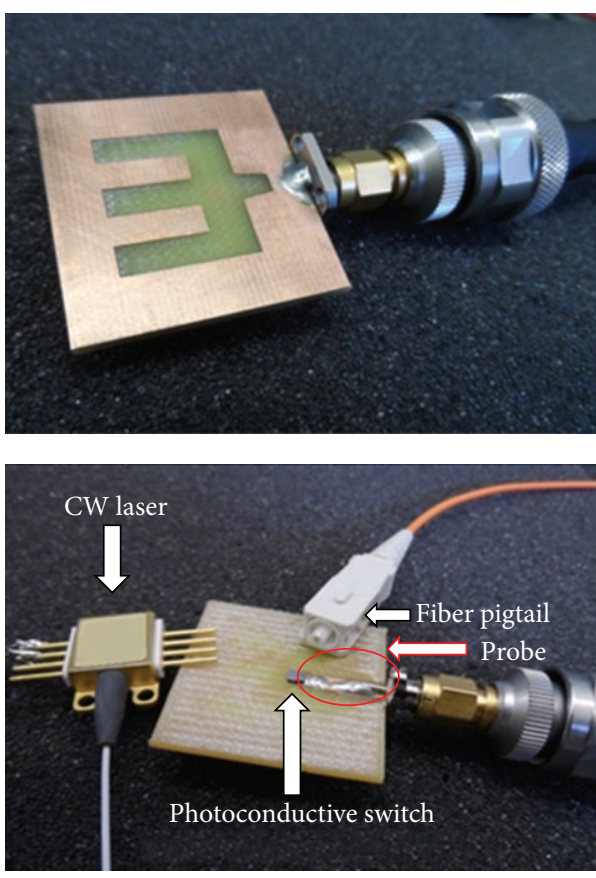

FigURE 2: Fabricated E-antenna.

the proposed architecture can significantly improve the network performance.

The paper is organized as follows. The E-antenna design is described in Section 2, and its main numerical and experimental results are presented in Section 3. Section 4 reports the fabrication of antenna arrays based on E-shaped elements. Finally, the conclusions and final remarks are presented in Section 5 .

\section{E-Antenna Design}

Patch antennas have been significantly exploited in the past due to their low-profile structure and compatibility with being embedded in handheld wireless devices. They also provide other advantages, such as good radiation efficiency, simple manufacturing, low cost, ease of integration with microwave integrated circuits, light weight, low volume, and the possibility of being conformed to the host surface. Particularly, Khidre et al. recently reported results on a circular polarization reconfigurable E-shaped patch antenna using two PIN diodes [10]. Our proposed patch antenna is based on an E-shaped printed slot fabricated in a lowcost fiberglass dielectric substrate, as presented in Figure 2. It is based on electromagnetic coupling ensured by a printed probe located in the bottom plane of the antenna structure, as shown in the bottom part of Figure 2. This figure also displays the other components used in the experiments, namely, a photoconductive switch made from a $2 \times 1 \mathrm{~mm}$ intrinsic silicon die, a $2 \mathrm{~W} C W$ laser at $808 \mathrm{~nm}$, and a fiber pigtail for coupling light from the CW laser to the photoconductive switch. 


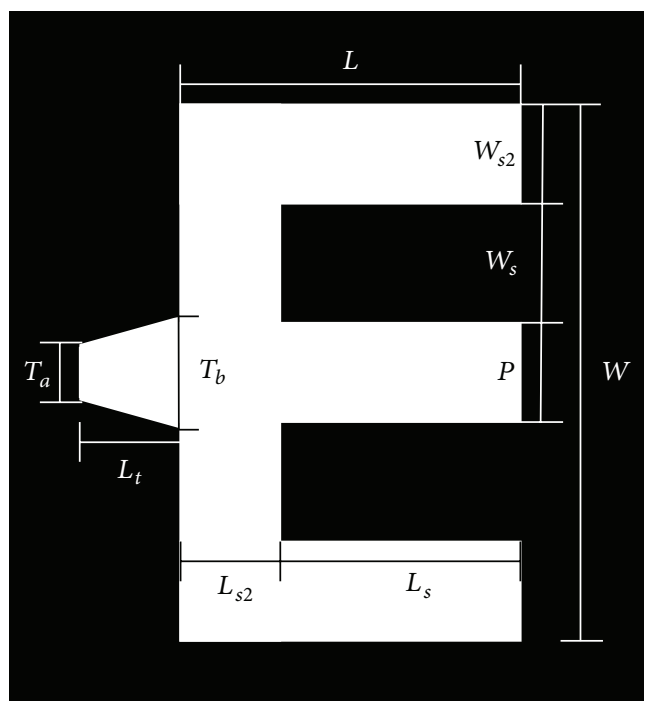

(a) Main parameters

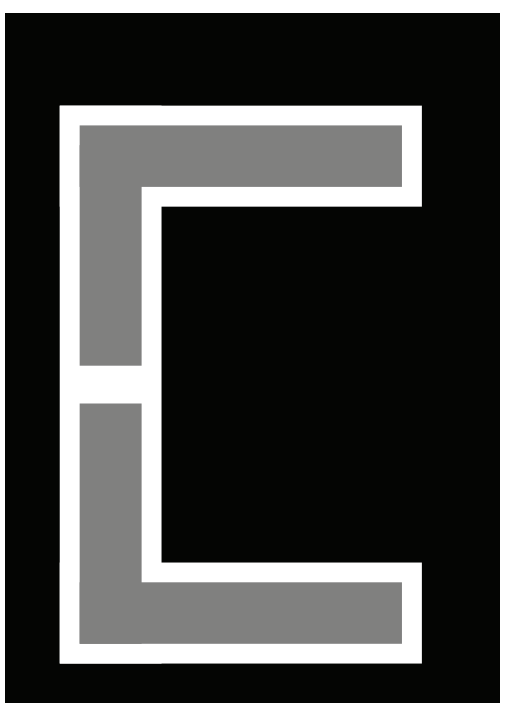

$2.4 \mathrm{GHz}$

$5.8 \mathrm{GHz}$

(b) Dual-band explanation

FIGURE 3: E-antenna model.

The E-antenna has been designed to provide two frequency bands $(2.4 \mathrm{GHz}$ and $5 \mathrm{GHz})$. Its entire structure, as detailed in Figure 3, has been optimized by manipulating the trapezoidal dimensions, $W$ and $L$, and varying the Eshaped parameters, $W_{s}, W_{s 2}$, and $P$. The highest frequency band is determined by the "C-structure," the white region in Figure 3(b), which is approximately equal to its guided wavelength. The two gray arms from Figure 3(b), the " $L$ structures," individually correspond to the guided wavelength of the central frequency from the lower band.

The prototype parameters are as follows: $W=20.48 \mathrm{~mm}$, $L=17.65 \mathrm{~mm}, W_{s}=W_{s 2}=4.1 \mathrm{~mm}, L_{s}=12 \mathrm{~mm}, L_{s 2}=$ $5.65 \mathrm{~mm}, L_{t}=9.5 \mathrm{~mm}, T_{a}=3 \mathrm{~mm}$, and $T_{b}=4.31 \mathrm{~mm}$. An intrinsic silicon photoconductive switch has been fixed to the printed probe, which enables reconfiguring of the antenna electromagnetic properties as a function of optical power. A fiber pigtail has been used to illuminate the silicon die with light from the CW laser.

The antenna optical control can be explained as follows. The probe is extremely important for antenna impedance matching, and the photoconductive switch, which has been fixed to the antenna, can manage its length. For the off-state, the die assumes the highest resistance, which is approximately $6000 \Omega$ [11]; therefore, the probe length is limited to the printed metallic line. As soon as the photoconductive switch is illuminated, the silicon changes from an insulator state to a near conducting state by creating electron-hole pairs. In this way, the current starts to increase because the switch resistance is reduced and, consequently, the silicon wafer becomes able to conduct. Its minimum value for the onstate is approximately $118 \Omega$ [6]. The incident photons must have enough energy to move electrons from the valence to the conduction band. Light in the near-infrared region is adequate for this process as it strikes a balance between the absorption coefficient and the light penetration depths [12], which are inversely proportional to each other and related to the light wavelength. Therefore, the switch conductivity can be managed by controlling the optical incident power. In this way, the probe length can be modified, and, consequently, the antenna frequency response can be reconfigured as a function of the laser current. In the proposed antenna, the CW laser is used to transpose the $1.12 \mathrm{eV}$ bandgap between the valence and conduction bands from the intrinsic silicon die.

\section{E-Antenna Results}

ANSYS HFSS numerical simulations and experimental characterization are used to analyze the antenna. Initially, the reflection coefficient $\left(S_{11}\right)$ was evaluated for the two main states of the photoconductive switch: the off-state (when there is an absence of light on it) and the on-state (when it is illuminated by the $808 \mathrm{~nm}$ laser). Figure 4 reports comparisons between the numerical and experimental results for the off- and on-states, which present good agreement. When the switch is in the off-state, the antenna frequency response is much better for the $5 \mathrm{GHz}$ band, with the reflection coefficient reaching a minimum of -22 and $-40 \mathrm{~dB}$ in the 


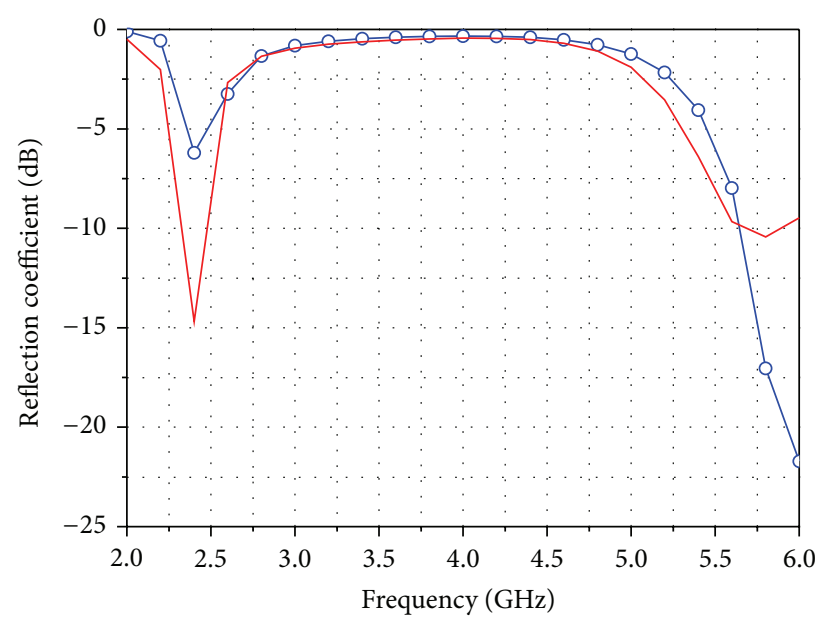

(a) Simulations

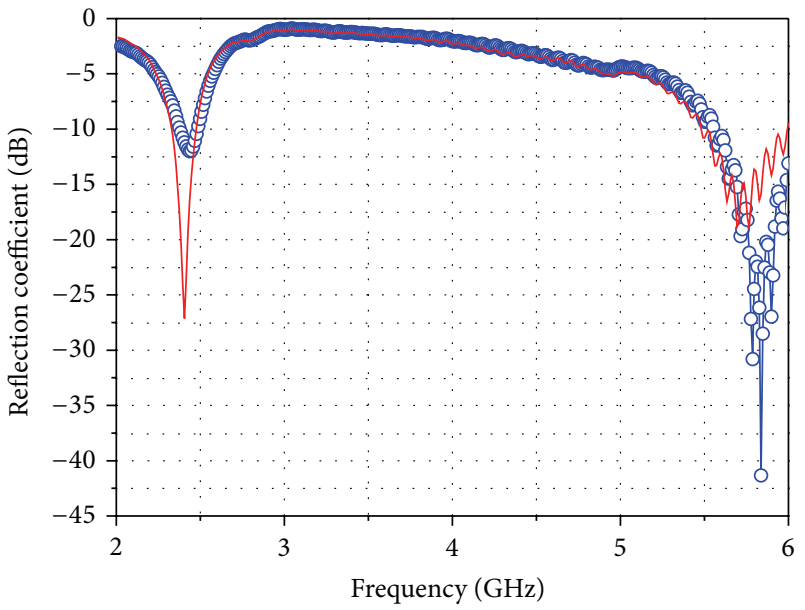

(b) Measurements

FIGURE 4: Reflection coefficient comparison as a function of the switch state: on-state (continuous red line); off-state (blue dashed line with circles).

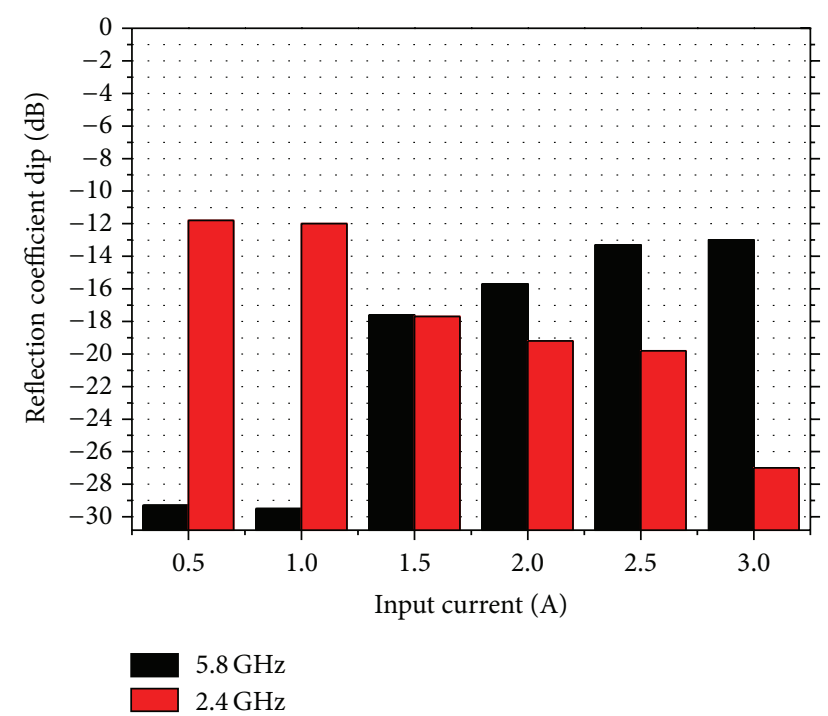

FIGURE 5: Measurement of reflection coefficient dips as a function of laser current.

simulation and experiment, respectively. For the $2.4 \mathrm{GHz}$ band, it is -6 and $-10 \mathrm{~dB}$, respectively. As soon as the photoconductive switch is illuminated by the $808 \mathrm{~nm}$ CW laser, an incident power into the switch of approximately $300 \mathrm{~mW}$, the antenna reconfigures its frequency response to significantly improve the reflection coefficient in the $2.4 \mathrm{GHz}$ band. In this state, the $2.4 \mathrm{GHz}$ band has a measured bandwidth of $8.3 \%$ and dip of $-27 \mathrm{~dB}$; the $5 \mathrm{GHz}$ band also has good performance, with a measured bandwidth of $8.7 \%$ and dip of $-19 \mathrm{~dB}$. It is clear that the antenna frequency response can be efficiently reconfigured through the two ISM bands in accordance with the system requirements.
The antenna frequency response of the OCRA can also be evaluated by impedance matching. An enhancement of the antenna input impedance $\left(Z_{\text {in }}\right)$ at $2.4 \mathrm{GHz},[41.31-$ $26.37 j] \Omega$ and [49.57 - 3.47j] $\Omega$ for the off- and on-states, respectively, was obtained. Its imaginary part is significantly reduced, and the real part becomes very close to the ideal value $(50 \Omega)$. For these two reasons, the impedance matching improves significantly for this frequency. For $5.8 \mathrm{GHz}$, the $S_{11}$ measurements for both switch states are very close to the central point of the Smith chart because their input impedances are $[56.87-0.41 j] \Omega$ and $[68.25+8.24 j] \Omega$ for the off- and on-states, respectively. These results reaffirm the successful implementation of the OCRA.

The switch conductivity strongly depends on the number of generated electron-hole pairs and, consequently, the level of incident optical power. In accordance with [6], its conductivity is only $52 \mathrm{~S} / \mathrm{m}$ for the off-state and can be increased to $622 \mathrm{~S} / \mathrm{m}$ by illuminating it at $212 \mathrm{~mW}$. An experimental analysis of the $S_{11}$ dips (minimum points) has been performed to illustrate this effect; the obtained results for the two main ISM bands are presented in Figure 5. The higher the laser current, the better the dip at $2.4 \mathrm{GHz}$, which improves from -12 to $-27 \mathrm{~dB}$. The physical mechanism behind this phenomenon is the increase in the probe length. Alternatively, the dip at $5.8 \mathrm{GHz}$ becomes worse as the optical power is increased due to the same reason. Additionally, it is important to highlight that there is a trade-off point approximately $1.5 \mathrm{~A}$.

The software ANSYS HFSS has also been used to numerically analyze the E-shaped antenna radiation pattern. The simulated tridimensional patterns at 2.4 and $5.8 \mathrm{GHz}$ for the off-state are shown in Figure 6. As expected, the gain for the $5.8 \mathrm{GHz}$ band $(G=5.30 \mathrm{dBi})$ is higher than that for the $2.4 \mathrm{GHz}$ band $(G=3.29 \mathrm{dBi})$ because the E-antenna operates differently for the two frequency bands. The lower band is determined by the trapezoidal dimensions, which approximately correspond to the guided wavelength for this band. For the higher band, the E-shaped antenna operates 


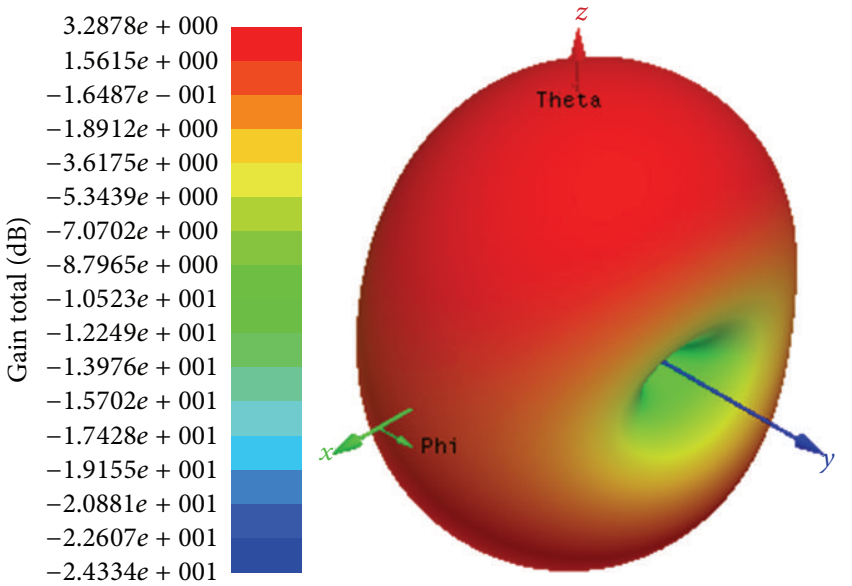

(a) $2.4 \mathrm{GHz}$

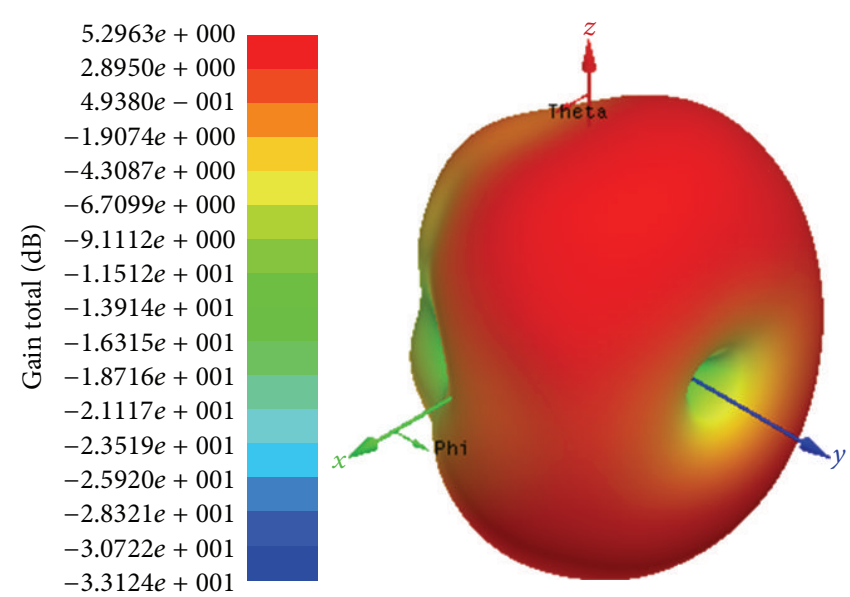

(b) $5.8 \mathrm{GHz}$

FIGURE 6: Simulation of the E-shaped antenna radiation patterns for the off-state.

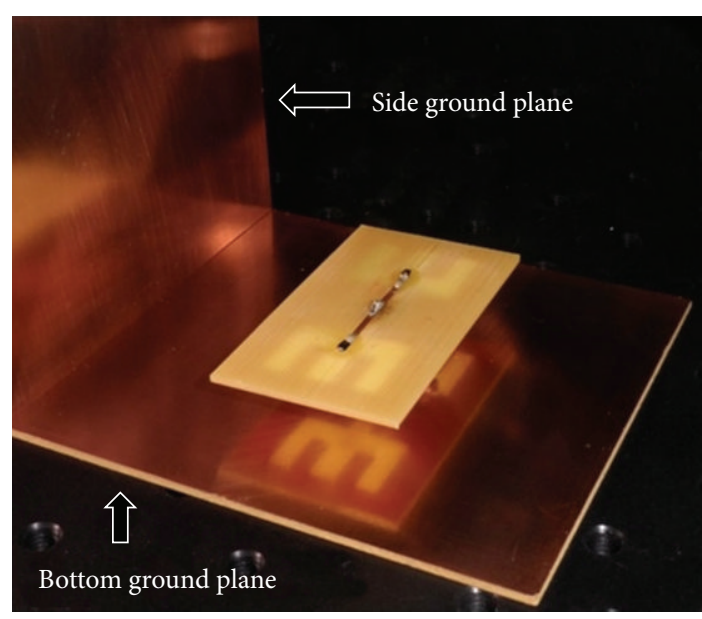

FIgURE 7: Photograph of antenna array with a corner reflector.

based on two arms that are as long as its guided wavelength. For this reason, its gain is higher than the previous one.

\section{Antenna Arrays}

Three different configurations of antenna arrays based on Eshaped elements have been constructed to increase the directivity. The following configurations have been numerically and experimentally evaluated:

configuration I: one E-shaped antenna;

configuration II: array with two E-shaped elements;

configuration III: array with two E-shaped elements and a bottom ground plane;

configuration IV: array with two E-shaped elements and a dihedral corner reflector based on the bottom and side ground planes.
A photograph of configuration IV is displayed in Figure 7. For this case, two photoconductive switches were used, one for each E-shaped element. The angle between the $10 \times 10 \mathrm{~cm}$ metallic reflector plates is 90 degrees. The radiation pattern of all configurations has been measured in the semianechoic chamber of our institute and compared with the results of the numerical simulations.

Figure 8 presents a comparison of the simulated and measured radiation patterns from configuration III at $5.8 \mathrm{GHz}$ for the off-state. There is an excellent agreement between simulations and measurements. The azimuth pattern is omnidirectional, whereas the elevation pattern presents two lobes, as in the pattern of one E-shaped element (configuration I) presented in the previous section. The main difference between configurations I and III is the gain magnitude, which increased from 5.30 to $10 \mathrm{dBi}$, respectively. As expected, the radiation pattern and gain from configuration III at $5.8 \mathrm{GHz}$ for the off- and on-states are very similar. On the other hand, the gain from configuration III at $2.4 \mathrm{GHz}$ can be improved when the photoconductive switch is illuminated by the $808 \mathrm{~nm}$ laser, as reported in Figure 9. The simulated and measured gain for the on-state is approximately $5.3 \mathrm{dBi}$ in this case. Furthermore, the elevation pattern from configuration IV for both switch states was also measured and is displayed in Figure 10. The effect of the antenna optical control is similar to that from configuration III, that is, a gain improvement for $2.4 \mathrm{GHz}$ and no changes for $5.8 \mathrm{GHz}$.

Finally, an analysis of the gain improvement was performed for all configurations at 2.4 and $5.8 \mathrm{GHz}$, as shown in Figure 11. It is clear that the directivity can be significantly improved by using the proposed configurations. The spacing between the array elements has been numerically optimized for $5.8 \mathrm{GHz}$. For this reason, the gain increase is higher for this frequency than that for $2.4 \mathrm{GHz}$. The gain at $2.4 \mathrm{GHz}$ increased from $2.7 \mathrm{dBi}$ from configuration I to $6.8 \mathrm{dBi}$ by using configuration IV. The measured gains at $5.8 \mathrm{GHz}$ were $5.1,7.5,10$, and $12.5 \mathrm{dBi}$ for configurations I, II, III, and IV, respectively. As in the previous sections, there is excellent 


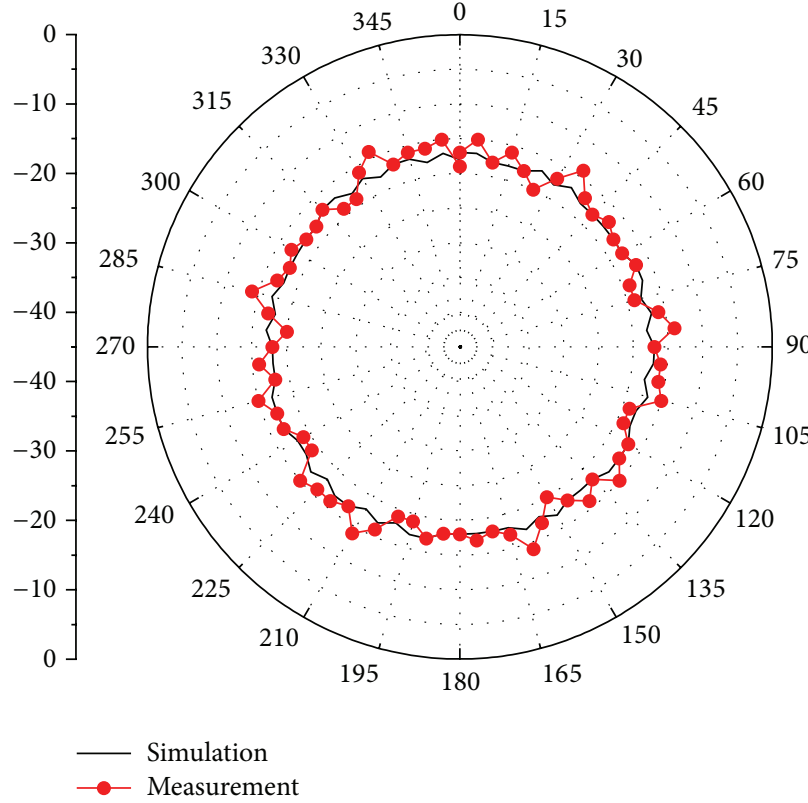

(a) Azimuth pattern

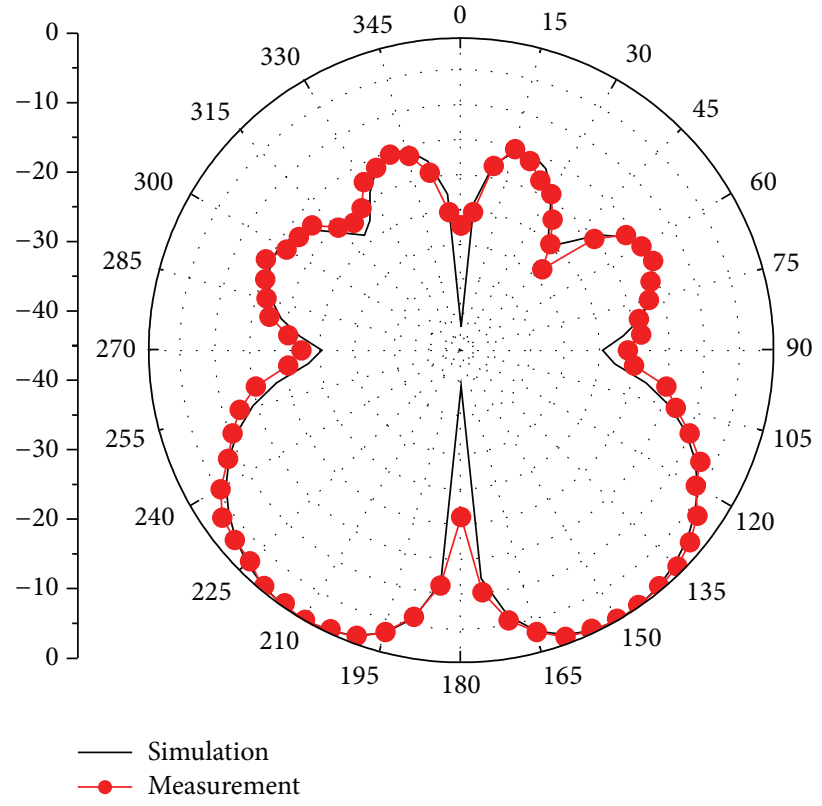

(b) Elevation pattern

FIGURE 8: Simulated and measured radiation patterns from configuration III at $5.8 \mathrm{GHz}$.

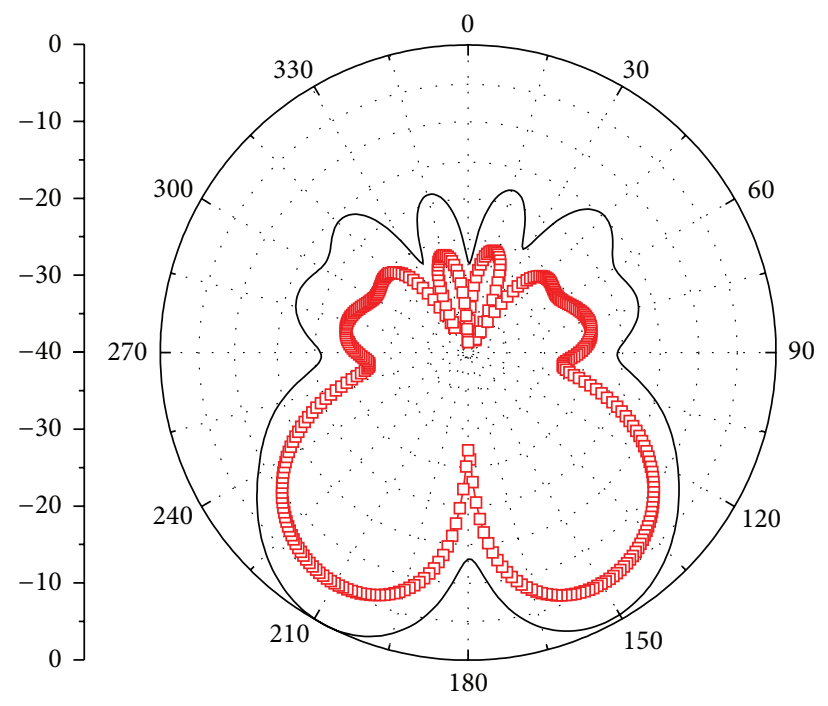

- On-state

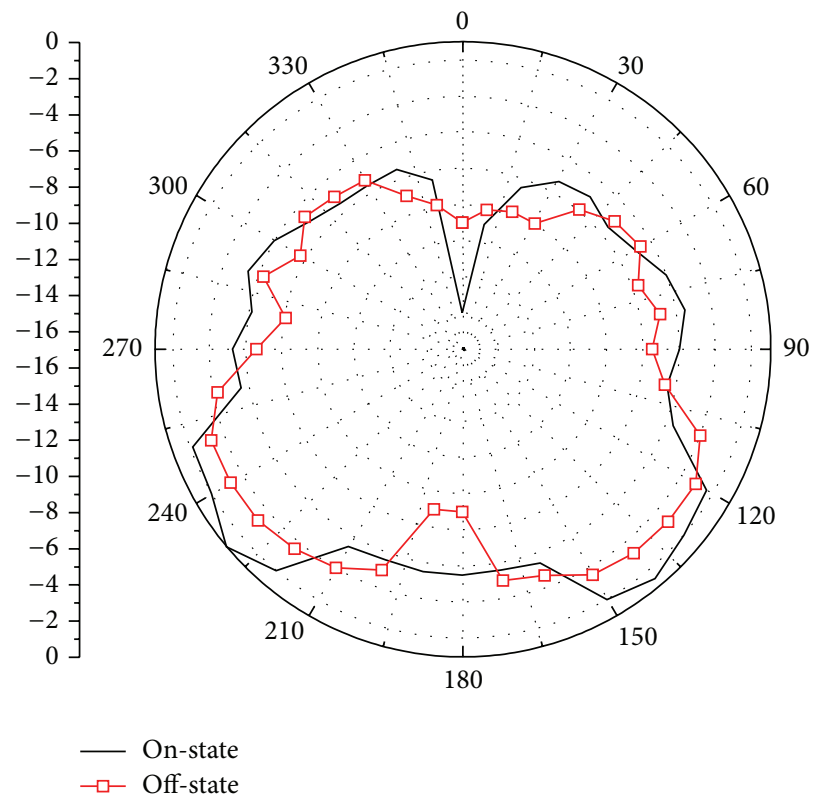

(b) Measurements

(a) Simulations

FIGURE 9: Elevation patterns from configuration III at $2.4 \mathrm{GHz}$.

agreement between HFSS simulations and experimental results.

\section{Conclusions}

Low-cost, frequency-agile, and high-gain optically controlled reconfigurable arrays based on E-shaped elements have been successfully proposed and fabricated. Reconfigurable bandwidths over two ISM bands have been reported. Comparisons between the simulations and measurements of the reflection coefficient and radiation patterns have been shown to be in excellent agreement. The array composed of two E-shaped elements and a dihedral corner reflector provides measured gains of 6.8 and $12.5 \mathrm{dBi}$ at 2.4 and $5.8 \mathrm{GHz}$, respectively. 

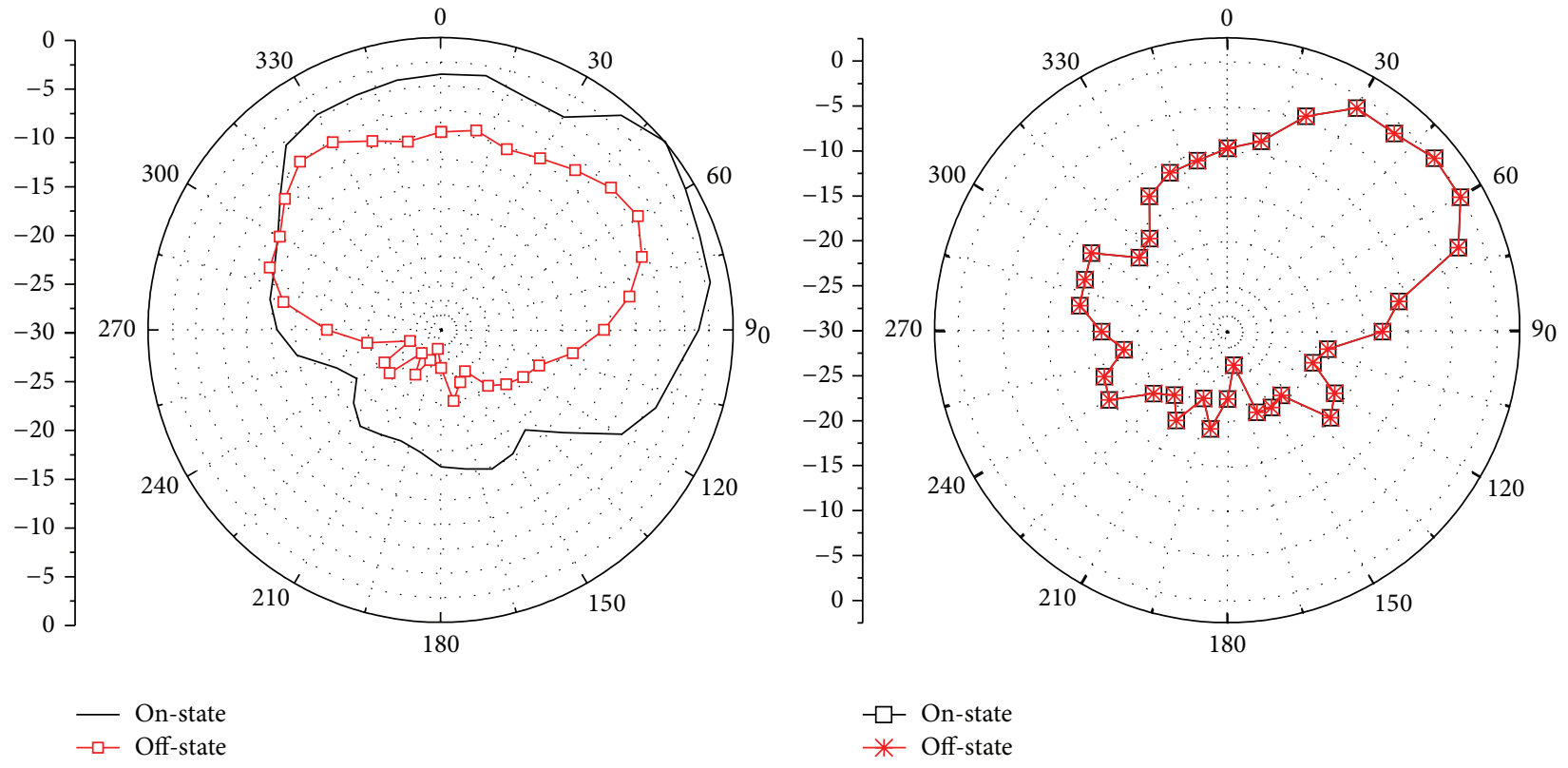

(a) $2.4 \mathrm{GHz}$

(b) $5.8 \mathrm{GHz}$

FIGURE 10: Measured elevation patterns from configuration IV.

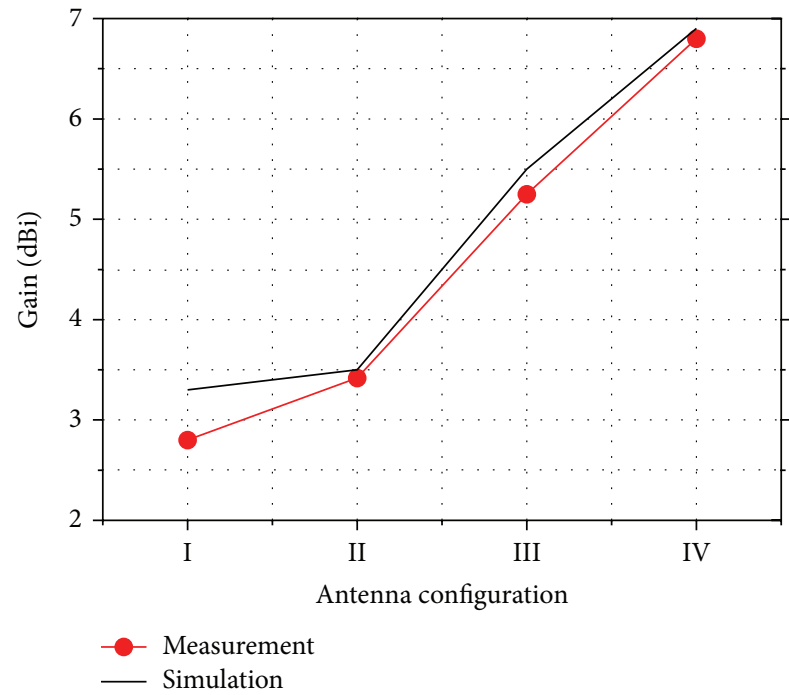

(a) $2.4 \mathrm{GHz}$

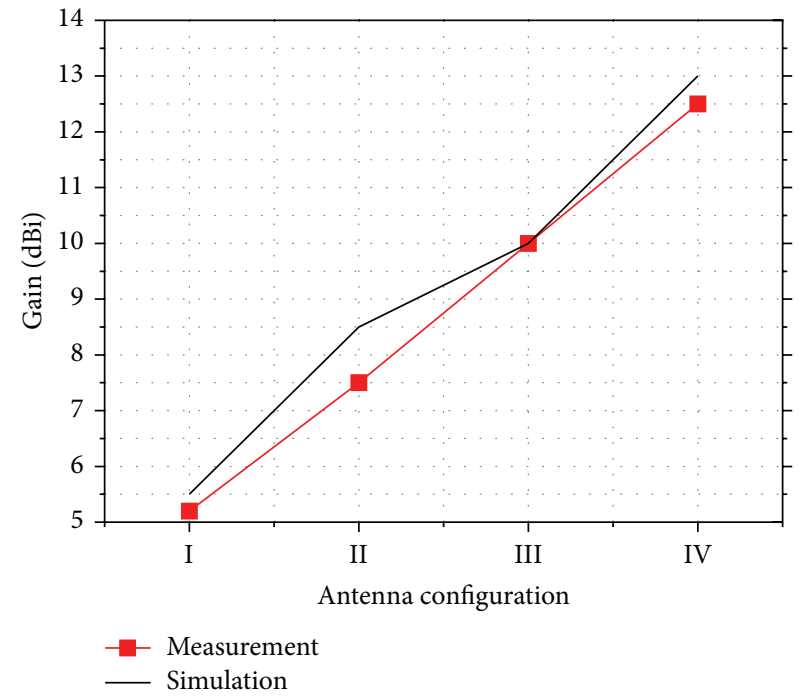

(b) $5.8 \mathrm{GHz}$

FIGURE 11: Gain analyses for all configurations.

Experimental results have demonstrated that a laser at the mid-infrared region can remotely control the antenna frequency response. This outstanding feature allows for the creation of smart, cognitive, and agile RF devices that can both sense the surrounding RF environment and communicate at the same time using different frequency bands.

\section{Conflict of Interests}

The authors declare that there is no conflict of interests regarding the publication of this paper.

\section{Acknowledgments}

The authors thank the financial support of CNPq, CAPES, MCTI, FAPEMIG, FINATEL, ESSS-ANSYS, Bradar, Prysmian-Draka, and Huber-Suhner and the technical support of TIM.

\section{References}

[1] J. Mitola III and G. Q. Maguire Jr., "Cognitive radio: making software radios more personal," IEEE Personal Communications, vol. 6, no. 4, pp. 13-18, 1999. 
[2] E. Larsson and M. Skoglund, "Cognitive radio in a frequencyplanned environment: some basic limits," IEEE Transactions on Wireless Communications, vol. 7, no. 12, pp. 4800-4806, 2008.

[3] A. J. Seeds and K. J. Williams, "Microwave photonics," Journal of Lightwave Technology, vol. 24, no. 12, pp. 4628-4641, 2006.

[4] D. G. Lona, R. M. Assumpção, O. C. Branquinho, M. L. F. Abbade, H. E. Hernandez Figueroa, and S. Arismar Cerqueira Jr., "Implementation and performance investigation of radioover-fiber systems in wireless sensor networks," Microwave and Optical Technology Letters, vol. 54, pp. 2669-2675, 2012.

[5] C. G. Christodoulou, Y. Tawk, S. A. Lane, and S. R. Erwin, "Reconfigurable antennas for wireless and space applications," Proceedings of the IEEE, vol. 100, no. 7, pp. 2250-2261, 2012.

[6] Y. Tawk, A. R. Albrecht, S. Hemmady, G. Balakrishnan, and C. G. Christodoulou, "Optically pumped frequency reconfigurable antenna design," IEEE Antennas and Wireless Propagation Letters, vol. 9, pp. 280-283, 2010.

[7] Y. Tawk, J. Costantine, S. Hemmady, G. Balakrishnan, K. Avery, and C. G. Christodoulou, "Demonstration of a cognitive radio front end using an optically pumped reconfigurable antenna system (OPRAS)," IEEE Transactions on Antennas and Propagation, vol. 60, no. 2, pp. 1075-1083, 2012.

[8] C. J. Panagamuwa, A. Chauraya, and J. C. Vardaxoglou, "Frequency and beam reconfigurable antenna using photoconducting switches," IEEE Transactions on Antennas and Propagation, vol. 54, no. 2, pp. 449-454, 2006.

[9] E. R. Neto, M. A. F. Casaroli, J. R. G. da Rosa et al., "Dynamic spectral sensing and resource allocation in optical-wireless network," in SDR-WInnComm, Schaumburg, Ill, USA, 2014.

[10] A. Khidre, Kai-Fong Lee, F. Yang, and A. Z. Elsherbeni, "Circular polarization reconfigurable wideband E-shaped patch antenna for wireless applications," IEEE Transactions on Antennas and Propagation, vol. 61, no. 2, pp. 960-964, 2013.

[11] E. K. Kowalczuk, C. J. Panagamuwa, R. D. Seager, and J. C. Vardaxoglou, "Characterising the linearity of an optically controlled photoconductive microwave switch," in Proceedings of the 6th Loughborough Antennas and Propagation Conference (LAPC '10), pp. 597-600, Loughborough, UK, November 2010.

[12] B. E. A. Saleh and M. C. Teich, Fundamentals of Photonics, Wiley, Haboken, NJ, USA, 2007. 

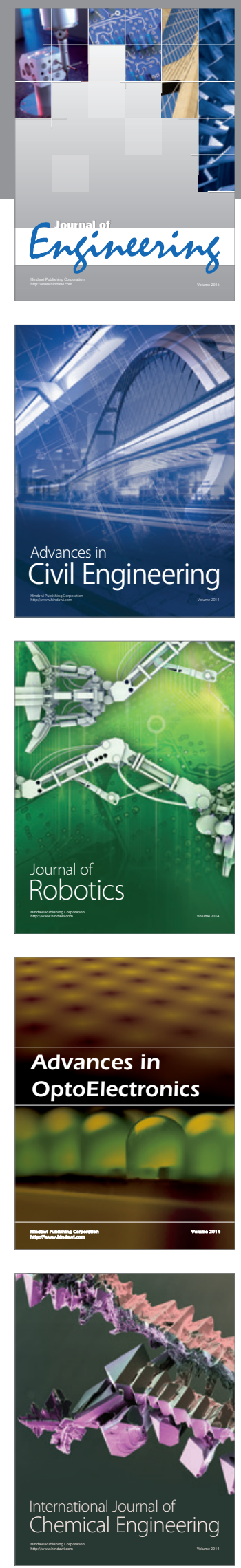

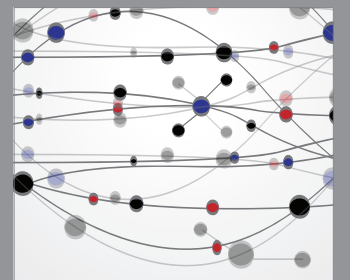

The Scientific World Journal
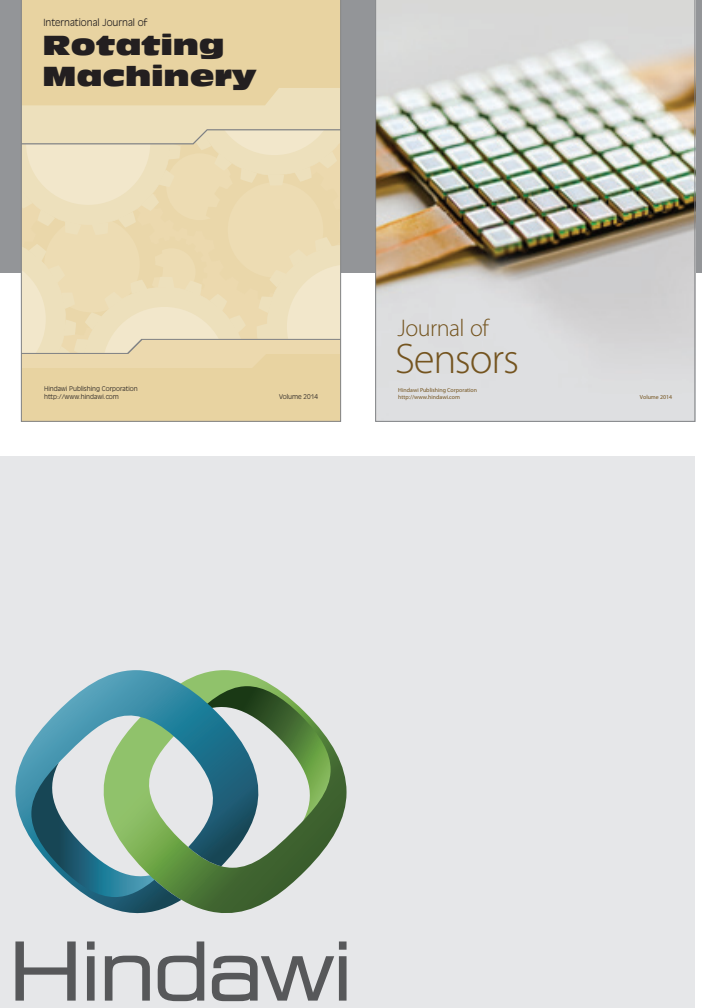

Submit your manuscripts at http://www.hindawi.com
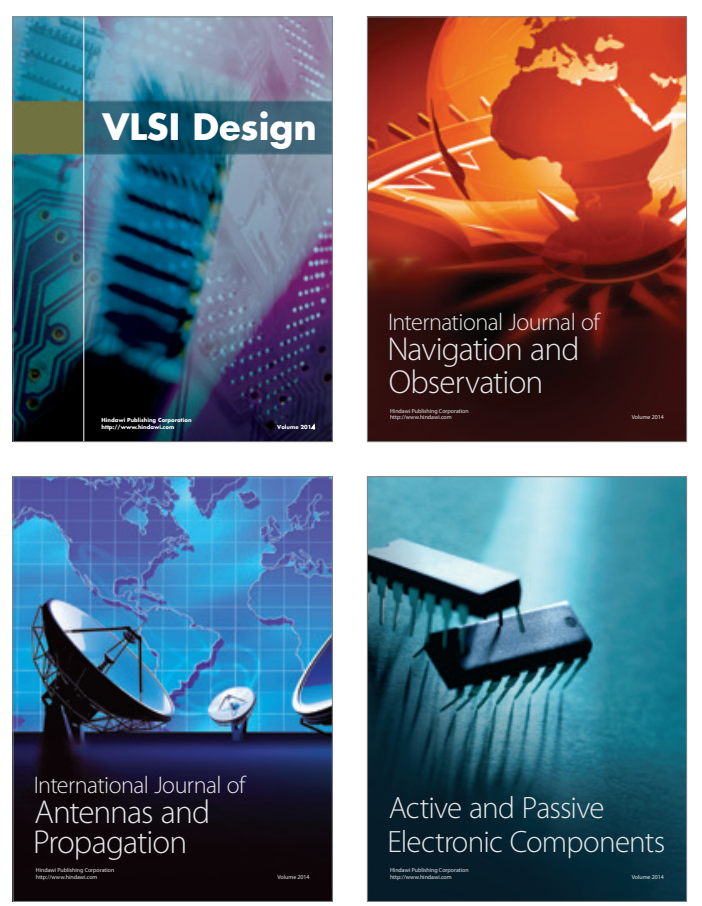
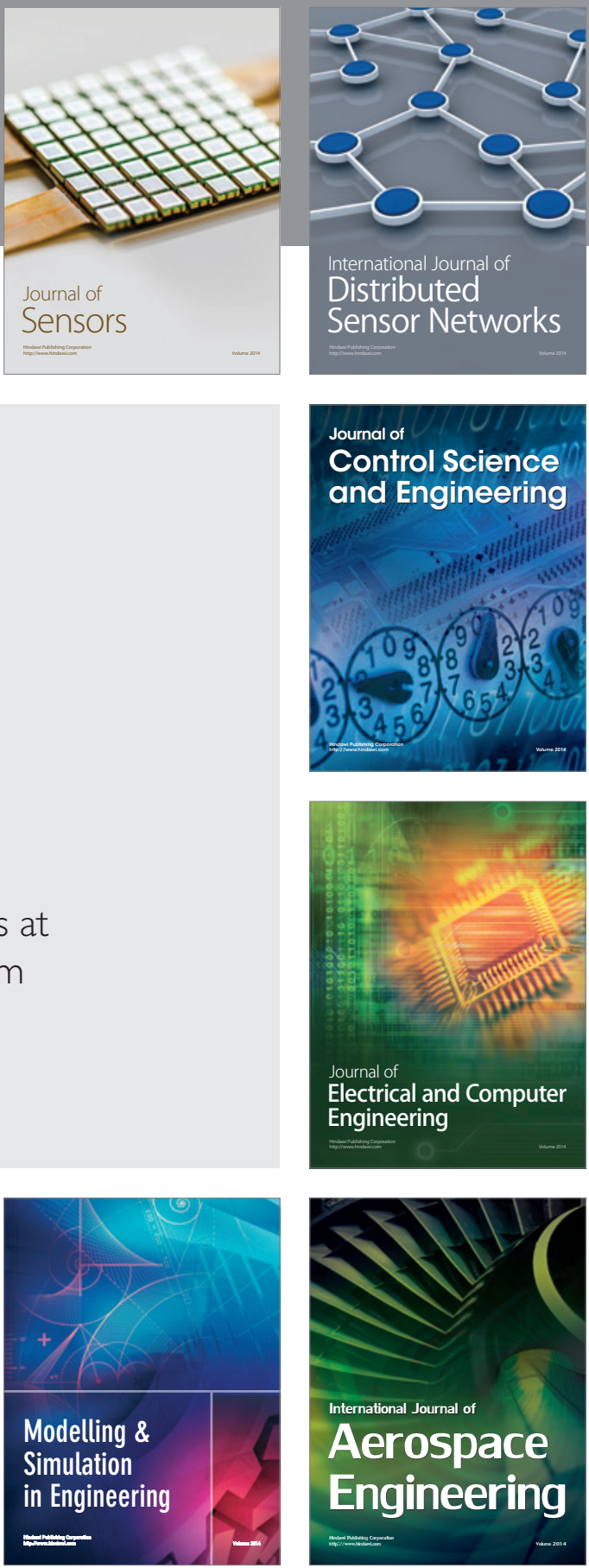

Journal of

Control Science

and Engineering
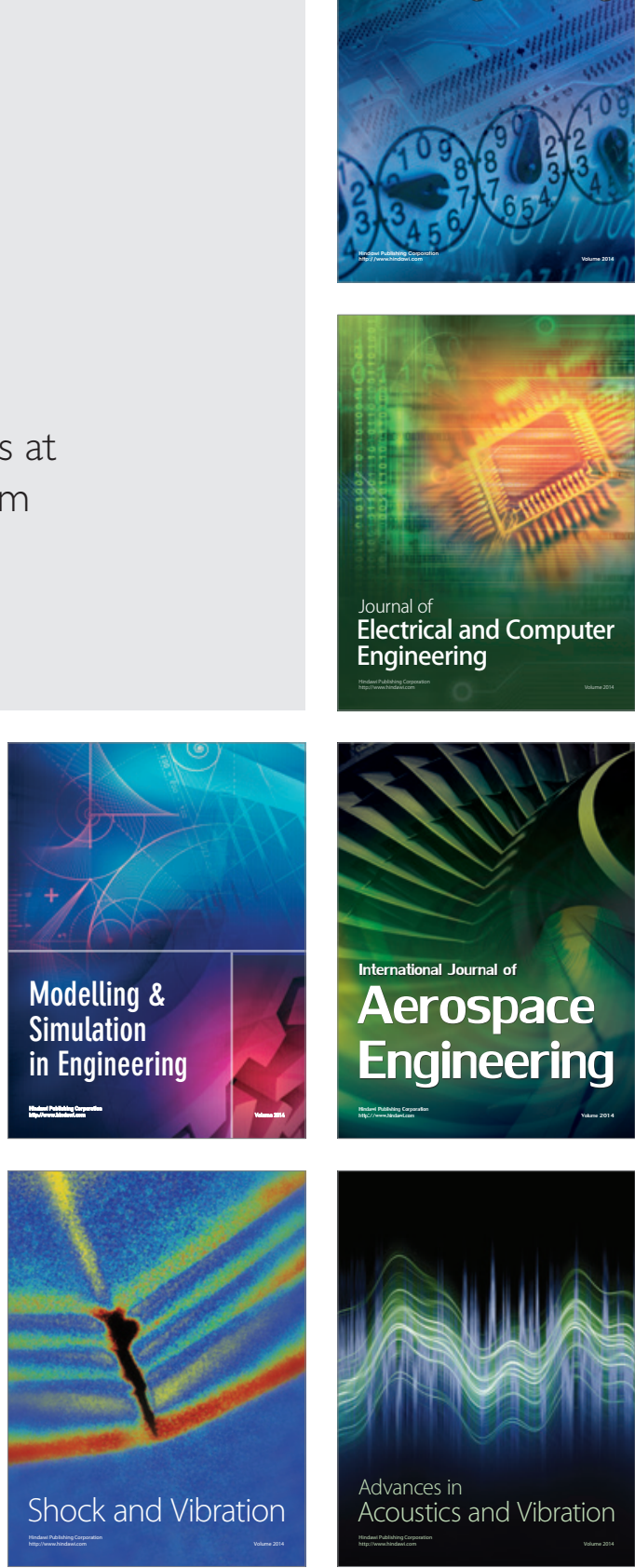